\title{
Development and use of a computer program to detect potentially inappropriate prescribing in older adults residing in Canadian long-term care facilities
}

\author{
Alexandra Papaioannou*1, Michel Bedard ${ }^{2}$, Glenda Campbell ${ }^{3}$, \\ Sacha Dubois ${ }^{4}$, Nicole Ferko ${ }^{5}$, George Heckman ${ }^{6}$ and Norman Flett ${ }^{7}$
}

Address: ${ }^{1}$ Division of Geriatric Medicine, McMaster University Hamilton, Ontario, Canada, ${ }^{2}$ Lakehead Psychiatric Hospital, Thunderbay, Ontario, Canada, ${ }^{3}$ Medical Pharmacies Ltd. Pickering Ontario, Canada, ${ }^{4}$ Department of Clinical Epidemiology and Biostatistics, McMaster University Hamilton, Ontario, Canada, ${ }^{5}$ Department of Clinical Health Sciences, McMaster University Hamilton, Ontario, Canada, ${ }^{6}$ Department of Medicine, McMaster University Hamilton, Ontario, Canada and ${ }^{7}$ St. Joseph's Villa Dundas, Ontario, Canada

E-mail: Alexandra Papaioannou* - papaioannou@hhsc.ca; Michel Bedard - michel.bedard@lakeheadu.ca;

Glenda Campbell - ecampbell49@cogeco.ca; Sacha Dubois - sacha.dubois@hrcc.on.ca; Nicole Ferko - ferkonc@mcmaster.ca;

George Heckman - heck0@sympatico.ca; Norman Flett - nflett@sjv.on.ca

${ }^{*}$ Corresponding author

Published: 14 October 2002

Received: 4 July 2002

BMC Geriatrics 2002, 2:5

Accepted: 14 October 2002

This article is available from: http://www.biomedcentral.com/l47/-23/8/2/5

(C) 2002 Papaioannou et al; licensee BioMed Central Ltd. This article is published in Open Access: verbatim copying and redistribution of this article are permitted in all media for any purpose, provided this notice is preserved along with the article's original URL.

Keywords: inappropriate prescribing, long-term care, drug database, computer program, elderly

\begin{abstract}
Background: Inappropriate prescribing has been estimated to be as high as $40 \%$ in long-term care. The purpose of this study was to develop a computer program that identifies potentially inappropriate drug prescriptions and to test its reliability.

Methods: Potentially inappropriate prescriptions were identified based on modified McLeod guidelines. A database from one pharmacy servicing long-term care facilities in Ontario was utilized for this cross-sectional study. Prescription information was available for the 356 long-term care residents and included: the date the prescription was filled, the quantity of drug prescribed and the eight-digit drug identification number. The pharmacy database was linked to the computer-based program for targeting potential inappropriate prescriptions. The computer program's reliability was assessed by comparing its results to a manual search conducted by two independent research assistants.
\end{abstract}

Results: There was complete agreement between the computer and manual abstraction for the total number of potentially inappropriate prescriptions detected. In total, 83 potentially inappropriate prescriptions were identified. Fifty-three residents (14.9\%) received at least one potentially inappropriate prescription. Of those, twenty $(37.7 \%)$ received two potential inappropriate prescriptions and eight $(15.1 \%)$ received 3 or more potential inappropriate prescriptions. The most common potential inappropriate prescriptions were identified as longterm use of non-steroidal anti-inflammatory agents and tricyclic antidepressants with active metabolites.

Conclusion: A computer program can accurately and automatically detect inappropriate prescribing in residents of long-term care facilities. This tool may be used to identify potentially inappropriate drug combinations and educate health care professionals. 


\section{Background}

Nearly $25 \%$ of seniors over the age of 85 years reside in long-term care facilities in the United States [1]. These individuals are prescribed a greater number of medications than those residing in the community $[2,3]$. Inappropriate prescribing has been reported to be higher among elderly in long-term care, compared to community dwelling elderly, with estimates as high as 33\% to 40\% [4-6]. Multiple medical illnesses, functional impairment and cognitive deficits contribute to a higher risk of adverse drug reactions in the elderly residing in long-term care [7]. Adverse outcomes, including hospital admissions, increased cost and delirium have been associated with inappropriate prescribing [6,8-10].

Guidelines in both Canada [11] and the United States $[12,13]$ have been developed to guide prescribing for the elderly. Through a Delphi consensus process, McLeod and colleagues [11] proposed prescribing guidelines (with 38 recommendations) that incorporated Canadian practices and updated the Beers guidelines [12]. A shorter version of the McLeod guidelines was pilot tested in both acute and long-term care $[14,15]$. The studies required manual abstraction from patient charts.

Translation of evidence-based guidelines into practice may be facilitated by computerized instruments, which detect and flag potentially inappropriate prescriptions. Computerized tools in acute care have been shown to be effective in identifying adverse drug events and inappropriate drug dosing, and improving prescribing practices [16-18].

The goal of this study was to develop and test the reliability of a computer program, which would identify potentially inappropriate drug prescriptions, based on a modified version of the McLeod guidelines.

\section{Methods}

\section{Study design}

The administrative database from one pharmacy providing service to a long-term care facility was used in this cross-sectional study. The pharmacy provides service to approximately 200 facilities in Ontario. Complete prescription information was voluntarily provided for the month of November 2000 for 356 long-term care residents. The university ethics review board granted approval for the study. All patient, physician and facility identifying numbers were scrambled to preserve confidentiality.

The pharmacy database included the following information: the age and sex of the residents, their Ontario Health Insurance Plan number, and the prescriptions dispensed to each of the residents in the long term care facility. Drug prescription information included the date the prescrip- tion was filled, the dose and quantity of drug prescribed and the eight-digit drug identification number. Patient diagnoses were not available from the database. Linkage between the pharmacy database and the computer-based program developed to identify inappropriate prescriptions involved two steps. First, an anonymized version of the pharmacy database was exported into a Microsoft Excel format. Second, the Excel file was imported into a Microsoft Visual FoxPro table structure.

\section{Classification of inappropriate prescriptions}

There were 38 potentially inappropriate prescriptions defined using the McLeod guidelines [11]. Since disease information was not available from the pharmacy database, the inappropriate practices, which fell within the category "drug disease interactions", were modified, using drug prescriptions as surrogates for the disease state. For example, the McLeod guidelines described the prescription of a beta-blocker to an elderly individual with chronic obstructive pulmonary disease as potentially inappropriate. For the purpose of our study, we defined chronic obstructive pulmonary disease as any person who was prescribed a beta-adrenergic agonist or a bronchial anti-inflammatory agent. Thus, any resident who was prescribed one of these drugs and a beta-adrenergic blocking agent would have been flagged as having received a potentially inappropriate prescription. Long-term prescription was defined as prescription of a medication for 2 weeks or greater. Drug prescriptions were used as surrogates for the following disease conditions: chronic obstructive pulmonary disease, gout, glaucoma, benign prostate hyperplasia, heart failure, hypertension, peptic ulcer disease, and osteoarthritis.

Further, several assumptions were made that drugs were only used for the treatment of a specified disease. For example, for the practice: "Prescription of a thiazide diuretic to treat hypertension for patients with a history of gout", we assumed that a thiazide diuretic was always prescribed for hypertension. Similarly, for the practice: "Prescription of NSAIDS to treat osteoarthritis for patients with history of peptic ulcer", we assumed that NSAIDS were always prescribed for osteoarthritis.

Recommendations related to diseases that lacked a prescription drug marker and drugs that were no longer routinely prescribed were not applied to the study (Table 1). In total we used 27 of the original 38 recommendations that were modified to accommodate the pharmacy database.

Descriptive statistics were used to characterize the patient sample and the proportion of inappropriate prescriptions according to the McLeod guidelines. 
Table I: Inappropriate prescribing practices outlined in McLeod guidelines that were excluded in the current study

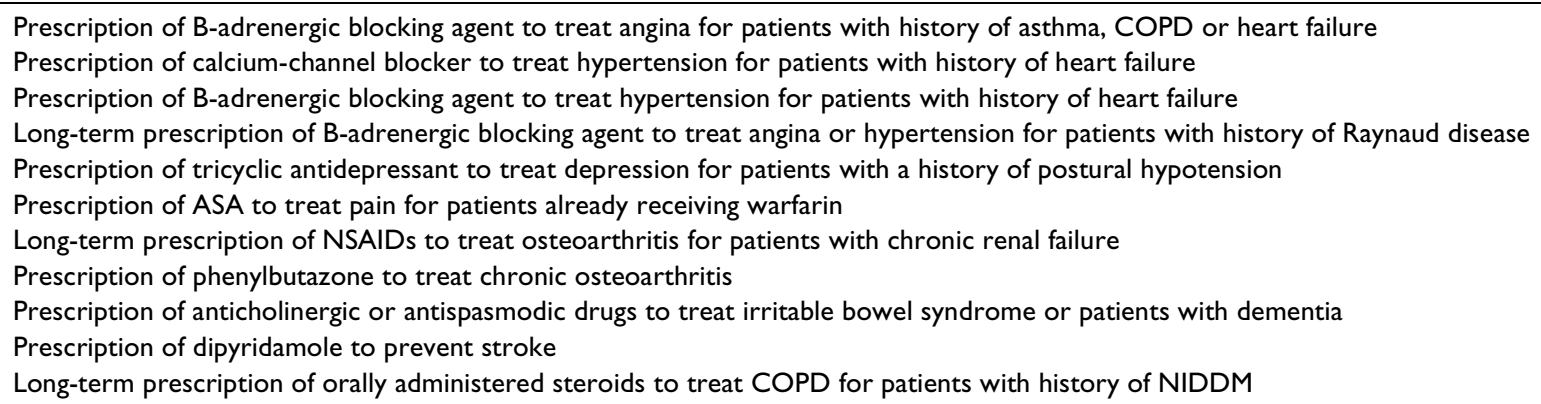

\section{Computer program}

Microsoft's Visual Fox Pro Version 6.0, a database development system was used to construct two algorithms: 1) a data set generator algorithm and 2) an inappropriate prescription detection algorithm. Both algorithms were developed using FoxPro's Visual basic and Structured Query Language components. The data set generator algorithm randomly generated two mock patient data sets containing both appropriate and inappropriate prescriptions. These data sets provided error free data where both manual abstractors and the computer programmer could refine the inappropriate prescription detection algorithm while remaining blind to the contents of the data set until after the inappropriate prescription detection was conducted. The data set generator algorithm contained four parameters: 1) the number of patients to be contained in the data set, 2) a minimum number of inappropriate prescriptions per patient, 3 ) the total number of prescriptions per patient, and 4) the class of inappropriate prescriptions to include.

The second algorithm, based on the relevant McLeod [11] recommendations, was used to detect inappropriate prescriptions. Drugs were identified using their drug identification numbers, as listed in the Ontario Drug Benefit Formulary for 2000. The inappropriate prescription detection algorithm was designed to flag both kinds of inappropriate prescriptions: those where a single drug indicated an inappropriate prescription and those where the combination of two drugs indicated an inappropriate prescription. Using this data set, the algorithm ran three scans of the patient's prescriptions. The first scan detected and flagged single inappropriate prescriptions while the second and third scan were used to detect combination inappropriate prescriptions. The second scan pulled out all possible prescription combinations per patient. Finally, the third scan extracted combination inappropriate prescriptions from the second scan's results.
The reliability of the computer program was assessed by comparing the results obtained with the program to a manual search conducted by two independent research assistants blinded to the results of the computer program. Each assistant reviewed each patient chart, provided from the pharmacy, independently with an abstraction form. They were provided with a brief training session on abstraction of variables by a pharmacist. A case-by-case comparison of the results was made from the computer and manual reviews. The following information was gathered by computer and manual abstraction: the number of potential inappropriate prescriptions per person, total potential inappropriate prescriptions, the type of potential inappropriate prescription and the combination of drugs prescribed inappropriately.

\section{Results}

Data was abstracted for 356 elderly residents, the majority of whom were Caucasian. There were 276 females and 80 males with an age range of 44 to 102 years and mean age (+standard deviation) of $84.2 \pm 8.1$ years. There was complete agreement between the computer and manual abstraction for the following: total number of potential inappropriate prescriptions detected, the number of potential inappropriate prescriptions per person, type of potential inappropriate prescription and the combination of drugs prescribed inappropriately.

In total, 83 potential inappropriate prescriptions were identified. Of all residents, fifty-three (14.9\%) received at least one potential inappropriate prescription. Of those, twenty $(37.7 \%)$ were identified as having at least two potential inappropriate prescriptions and eight (15.1\%) were identified as having 3 or more potential inappropriate prescriptions.

Table 2 describes the proportion of patients given inappropriate prescription according to classification by the McLeod guidelines. Non-steroidal anti-inflammatory agents and other analgesics comprised the majority of the 
Table 2: Number of Inappropriate Prescriptions (\%) according to classification by McLeod et al. (of original 38 recommendations).

Practice - McLeod Guideline
Our Definition using drug prescription surrogates

Inappropriate

Prescriptions n

(\%)
Total inappropriate practices - Cardiovascular diseases

$\gamma$ \#Prescription of B-adrenergic blocking agent to treat hypertension for patients with history of asthma or COPD

\#Prescription of disopyramide to treat atrial fibrillation $\gamma$ \#Prescription of thiazide diuretic to treat hypertension for patients with history of gout

Total inappropriate practices - Psychotropic Drugs

*\#Long-term prescription of long half-life benzodiazepine to treat insomnia

\# $\gamma$ Prescription of tricyclic antidepressants to treat depression for patients with history of glaucoma, benign prostatic hyperplasia, or heart block

*\#Long-term prescription of barbituate to treat insomnia

\#Prescription of selective serotonin reuptake inhibitor for patients already receiving a monoamine oxidase inhibitor to treat depression

*\#Long term prescription of triazolam to treat insomnia

\#Prescription of chlorpromazine to treat psychosis for patients with history of postural hypotension

\#Prescription of nylidrin, niacin, pentoxifylline to treat dementia \#Prescription of tricyclic antidepressants with active metabolites to treat depression

\#Prescription of methylphenidate to treat depression

Total inappropriate practices - NSAIDS and other analgesics

$\perp{ }^{\#}$ Prescription of NSAIDS to treat osteoarthritis for patients with a history of peptic ulcer

*Long term prescription of meperidine or pentazocine for pain $\perp$ \#Prescription of NSAIDS to treat osteoarthritis for patients taking warfarin

$\perp{ }^{\# *} \gamma^{+}$Long-term prescription of NSAIDS to treat osteoarthritis for patients with history of heart failure or hypertension

*\#Long term prescription of piroxicam, ketorolac, or mefenamic acid to treat pain

*\# $\gamma$ Long term prescription of indomethacin to treat gout

$\perp$ \#* Long term prescription of NSAIDS to treat osteoarthritis Total inappropriate practices - miscellaneous drugs \#Prescription of cimetidine to treat peptic ulcer for patients already receiving warfarin

\#Prescription of anticholinergic drugs to prevent extrapyramidal effects of antipsychotic drugs

\#*Long term prescription of diphyenoxylate to treat diarrhea \#Prescription of cyclobenzaprine or methocarbamol to treat muscle spasms
Prescription of B-adrenergic blocking agent with B-agonist or bronchial anti-inflammatory agent

Prescription of disopyramide

Prescription of thiazide diuretics with gout medication (e.g. allopurinol)

Long-term prescription of any long half-life benzodiazepine (e.g. valium)

Prescription of tricyclic antidepressant with glaucoma or prostate medication

Long-term prescription of any barbituate

Prescription of selective serotonin reuptake inhibitor for patients already receiving a monoamine oxidase inhibitor

Long-term prescription of triazolam

5 (6.0)

$0(0.0)$

I (I.2)

$30(36.1)$

$4(4.8)$

8 (13.6)

Prescription of chlorpromazine

Prescription of nylidrin, niacin or pentoxifylline

Prescription of tricyclic antidepressants

Prescription of methylphenidate

39 (47.0)

Prescription of NSAIDS with histamine 2 antagonists or proton pump inhibitors

Long term prescription of meperidine or pentazocine

Prescription of NSAIDS with warfarin

3 (3.6)

Prescription of NSAIDS with diuretics

Long term prescription of piroxicam, ketorolac or mefenamic acid

Long term prescription of indomethacin with gout medication (e.g. allopurinol)

Long term prescription of NSAIDS $18(21.7)$

8 (9.6)

I (1.2)

Prescription of cimetidine with warfarin

Prescription of anticholinergic drugs (e.g. congentin) with antipsychotic drugs (e.g. risperdal)

Long term prescription of diphenoxylate

$0(0.0)$

Prescription of cyclobenzaprin or methocarbamol

*Prescribed longer than 2 weeks, + Recommendations from McLeod guidelines grouped together, $\gamma$ Recommendations from McLeod guidelines that were modified by using drug prescriptions as surrogates for disease states \#Assumption made that drug only used in treatment of specified disease $\perp$ Excluding ASA (combination or alone) as an inappropriate prescription, including all other NSAIDS 
total number of potential inappropriate prescriptions $(\mathrm{n}=$ 39; 47\%). Non-steroidal anti-inflammatory agents were commonly prescribed in combination with diuretics indicating a history of heart failure or hypertension $(n=13$; $15.7 \%$ ) and in combination with histamine-2 antagonists or proton pump inhibitors for patients indicating a history of peptic ulcer disease $(n=5 ; 6.0 \%)$. The second most common class of inappropriate medications involved prescription of psychotropic drugs $(\mathrm{n}=30 ; 36.1 \%)$. For psychotropic drugs, prescription of a tricyclic antidepressant with active metabolite $(\mathrm{n}=17 ; 20.5 \%)$ or a tricyclic antidepressant in combination with glaucoma or prostate medication $(\mathrm{n}=8 ; 13.6 \%)$, were the most common potential inappropriate prescriptions in this category. Longterm prescription of long-acting benzodiazepines and barbituates were uncommon in this population. Of the total potential inappropriate prescriptions, $6(7.2 \%)$ were inappropriately prescriptions of anticholinergic drugs with antipsychotic medication.

\section{Discussion}

The computer program identified all of the potential inappropriate prescriptions as modified from the McLeod guidelines that were captured in the manual abstraction. Although definitions of inappropriate prescribing may need modification based on new research, the computer program can be easily adapted to incorporate necessary changes. The program can be incorporated into a userfriendly interface for clinicians, nurses, or health care workers to use. The advantage of the computer program currently studied is the increased accuracy and efficiency associated with direct linkage of pharmacy database information to the computer inappropriate prescribing program. This program would allow pharmacists, nurses and physicians to review prescribing practices and make evidence based decisions.

The computer program identified that $14.9 \%$ of residents had at least one potential inappropriate prescription according to the modified McLeod guidelines [11]. Many inappropriate prescribing practices, such as use of triazolam, chlorpromazine and methylphenidate, were not found. Some of the inappropriate contraindications are not absolute, such as the use of coumadin and aspirin and were excluded from the abstraction [19]. Our results are similar to a recent Canadian study, which used a chart review applying modified McLeod guidelines, and found the prevalence of inappropriate prescriptions to be $18.3 \%$ in longterm care. Gill et al found that the most common inappropriate prescribing practices were the use of anticholinergic drugs to manage antipsychotic side effects, tricyclic antidepressants with active metabolites, long-acting benzodiazepines, and non-steroidal anti-inflammatory drugs in patients with a history of peptic ulcer disease, hypertension or renal insufficiency [15].
The majority of potential inappropriate prescriptions in our study involved prescription of non-steroidal anti-inflammatory drugs and psychotropic drugs. Other studies have identified a higher rate of inappropriate prescribing. For example, a Canadian study using modified Beers criteria and McLeod guidelines found that $29.8 \%$ patients were prescribed an inappropriate drug in the community and institutions [20]. American studies identified that 33 to $40 \%$ of residents in long-term care received an inappropriate drug prescription as defined by modification of Beers 1997 criteria [4,6]. There may be a number of reasons why inappropriate prescribing was lower in our population. Consultant pharmacists involved with the population may be detecting potential inappropriate prescriptions early on, therefore reducing inappropriate prescribing and possibly adverse drug events. Another reducing factor may be the small number of prescribing primary care physicians who specialize in geriatrics in the long term care facility studied. A Canadian provincial database study concluded that as the number of prescribing physicians increases, the patient's risk of receiving an inappropriate prescription also increases [21]. Use of different guidelines for detecting inappropriate prescribing may have resulted in lower rates of inappropriate prescriptions in our population.

Easily identifying inappropriate prescribing practices is only one of the barriers to optimizing prescribing [22]. Studies have shown that interventions targeting inappropriate prescribing are effective in long-term care residents. One randomized controlled study of long-term care residents determined the effects of an educational program on non-steroidal anti-inflammatory drug use and clinical outcomes in long-term care. The intervention included the risks and benefits of non-steroidal anti-inflammatory drugs and an algorithm that substituted other therapies for the treatment of non-inflammatory musculoskeletal pain. The intervention was shown to be effective resulting in markedly decreased long-term prescription of non-steroidal anti-inflammatory drugs and increased acetaminophen use [23].

One important limitation of the study was that we did not abstract information on diagnoses from the charts in the long-term care facility. This information is often unavailable from long-term care charts. Prescription medication was used as a surrogate marker for disease states for several criteria, where some surrogates were better indicators than other surrogates for disease states. As a result, we may have somewhat overestimated the rate of inappropriate prescribing for particular categories, outlined here: 1) long-term prescription of barbituates to treat insomnia: regular use of barbituates may still occur in long term care for seizures, however this could not be distinguished from insomnia. 2) prescription of non-steroidal anti-inflam- 
matory agents in those with peptic ulcer: prescription of this medication may have also been used in those with gastroesophageal reflux disease, but it is only considered inappropriate for peptic ulcer. Also, it could not be distinguished whether non-steroidal anti-inflammatory agents were being prescribed with knowledge of a history of peptic ulcer or if gastroprotective agents (i.e. H2 antagonists, proton pump inhibitors) were being prescribed as a result of non-steroidal anti-inflammatory induced gastric toxicity. 3) prescription of tricyclic antidepressants to treat depression for patients with history of benign prostate hyperplasia: prescription of alpha blockers may have also been used for the treatment of hypertension. This limitation of surrogate markers will be resolved only when technological support will permit the electronic integration and manipulation of diagnostic data on individual patients. Another important limitation of the current computer program is lack of linkage to documentation of adverse events from their potentially inappropriate medication use. Modification of the McLeod guidelines is also another possible limitation of this study. However, a literature review of studies using the Beers criteria of inappropriate prescribing determined that most researchers modified the criteria and that inappropriate prescribing was observed despite methodological differences [24].

\section{Conclusions}

We found that a computer program can accurately and automatically detect potentially inappropriate prescriptions in residents of long-term care facilities. Although computerized tools have been used to identify inappropriate prescribing in acute care, this is a new and emerging area in the field of long-term care. As previously discussed, the magnitude of the problem of inappropriate prescribing is greater in long-term care (4-6) and the consequences greater due to the frail elderly population $(7,25-26)$. An automated, accurate computer program would be an inexpensive, efficient alternative to identifying inappropriate prescribing in long-term care elderly. In addition, it would facilitate knowledge transfer since it could be readily adapted to updated practice guidelines.

Future research will be directed at application of this program to a larger patient population in determining if this program can facilitate change in prescribing practices. Also, research will be aimed at determining if changes in prescribing will have an affect on patient outcomes.

\section{Abbreviations \\ NSAIDS - Nonsteroidal anti-inflammatory drugs}

\section{Competing interests}

None declared

\section{Authors' contributions}

AP conceived of the study, participated in the study design and drafted the manuscript. MB conceived of the study, participated in the study design and contributed to analysis. GC participated in the design and coordination of the study. SD designed the computer program and participated in the analysis. NF participated in the coordination and the analysis. GH participated in the study design. NF participated in the design and coordination.

All authors read and approved the final manuscript.

\section{Acknowledgements}

The authors wish to thank Jo-Anne Clarke, Marilyn Cakebread, Sandy Regalado, Patricia Miller, and Zuzana Malcova for their assistance with the study. We would especially like to thank Medical Pharmacies Group Inc for providing the administrative database for the study.

\section{References}

I. National Institute on Aging, US Department of Commerce, Economics and Statistics Administration, Bureau of the Census: Aging in the United States: past, present, future. Washington 1997

2. Chrischilles EA, Foley DJ, Wallace RB, Lemke JH, Semla TP, Hanlon JT, Glynn RJ, Ostfeld AM, Guralnik JM: Use of medications by persons 65 and over: data from the established populations for epidemiologic studies of the elderly. J Gerontol A Biol Sci Med Sci 1992, 47:MI37-I44

3. Ouslander JG: Medical care in the nursing home. JAMA 1989, 262(18):2582-2590

4. Dhall J, Larrat EP, Lapane KL: Use of potentially inappropriate drugs in nursing homes. Pharmacotherapy 2002, 22(I):88-96

5. Piecoro LT, Browning SR, Prince TS, Ranz TT, Scutchfield FD: A database analysis of potentially inappropriate drug use in an elderly medicaid population. Pharmacotherapy 2000, 20(2):221228

6. Beers MH, Ouslander JG, Fingold SF, Morgenstern H, Reuben DB, Rogers W, Zeffren MJ, Beck JC: Inappropriate medication prescribing in skilled nursing facilities. Ann Intern Med 1992, I 1 7:684-689

7. Avorn J, Gurwitz JH: The ambiguous relationship between age and adverse drug reactions. Ann Intern Med I 99|, I | 4:956-966

8. Schmader KE, Hanlon JT, Landsman PB, Samsa GP, Lewis IK, Weinberger M: Inappropriate prescribing and health outcomes in elderly veteran outpatients. Ann Pharmacother 1997, 3 I:529-533

9. Tafreshi MJ, Melby MJ, Kaback KR, Nord TC: Medication-related visits to the emergency department: a prospective study. Ann Pharmacother 1999, 33(12): 1252-1257

10. Rochon PA, Gurwitz JH: Optimizing drug treatment for elderly people: the prescribing cascade. BMJ 1997, 3 I 5(7 I I5):10961099

II. McLeod PJ, Huang AR, Tamblyn RM, Gayton DC: Defining inappropriate practices in prescribing for elderly people: a national consensus panel. Can Med Assoc J 1997, 156(3):385-39I

12. Beers MH, Ouslander JG, Rollingher I, Reuben DB, Brooks J, Beck JC: Explicit criteria for determining inappropriate medication use in nursing home residents. Arch Intern Med 1991, I 5 1:18251832

13. Beers $\mathrm{MH}$ : Explicit criteria for determining potentially inappropriate medication use by the elderly: an update. Arch Intern Med 1997, 157:1531-1536

14. Naugler CT, Brymer C, Stolee P, Arcese ZA: Development and validation of an improving prescribing in the elderly tool. Can J Clin Pharmacol 2000, 7(2): 103-107

15. Gill SS, Misiaszek BC, Brymer C: Improving prescribing in the elderly: a study in the long term care setting. Can J Clin Pharmacol 200I, 8(2):78-83

16. Walton R, Dovey S, Harvey E, Freemantle N: Computer support for determining drug dose: systematic review and metaanalysis. BMJ 1999, 3 I 8(7 I 89): $984-990$

17. McMullin ST, Reichley RM, Kahn MG, Claiborne KW, Bailey TC: Automated system for identifying potential dosage problems at 
a large university hospital. Am J Health Syst Pharm 1997, 54:545549

18. Goldberg DE, Baardsgaard G, Johnson MT, Jolowsky CM, Shepherd $M$, Peterson $C D$ : Computer-based program for identifying medication orders requiring dosage modification based on renal function. Am J Health Syst Pharm I991, 48:1965

19. Hirsh J: Guidelines for Antithrombotic Therapy. BC Decker Inc I991, II:40

20. Hogan DB, Ebly EM, Fung TS: Regional variations in use of potentially inappropriate medication by Canadian seniors participating in the Canadian Study of Health and Aging. Can J Clin Pharmacol 1995, 2(4):167-174

21. Tamblyn RM, McLeod PJ, Abrahamowicz M, Laprise R: Do too many cooks spoil the broth? Multiple physician involvement in medical management of elderly patients and potentially inappropriate drug combinations. Can Med Assoc J 1996, I54(8): $1177-1183$

22. Beers MH, Fingold SF, Ouslander JG: A computerized system for identifying and informing physicians about problematic drug use in nursing homes. I Med Syst 1992, 16(6):237-245

23. Stein MC, Griffin MR, Taylor JA, Pichert JW, Brandt KD, Ray WA: Educational program for nursing home physicians and staff to reduce use of non-steroidal anti-inflammatory drugs among nursing home residents. Medical Care 200I, 39(5):436-445

24. Aparasu RR, Mort JR: Inappropriate prescribing for the elderly: beers criteria-based review. Ann Pharmacother 2000, 34(3):33846

25. Ballard C, O'Brien J, James I, Mynt P, Lana M, Potkins D, Reichelt K, Lee L, Swann A, Fossey J: Quality of life for people with dementia living in residential and nursing home care: the impact of performance on activities of daily living, behavioural and psychological symptoms, language skills, and psychotropic drug use. Int Psychogeriatrics 200I, 13(I):93-106

26. McGrath AM, Jackson GA: Survey of neuroleptic prescribing in residents of nursing homes in Glasgow. BMJ 1996, 3 | 2:6 | I-6I2

\section{Pre-publication history}

The pre-publication history for this paper can be accessed here:

http://www.biomedcentral.com/1471-2318/2/5/prepub

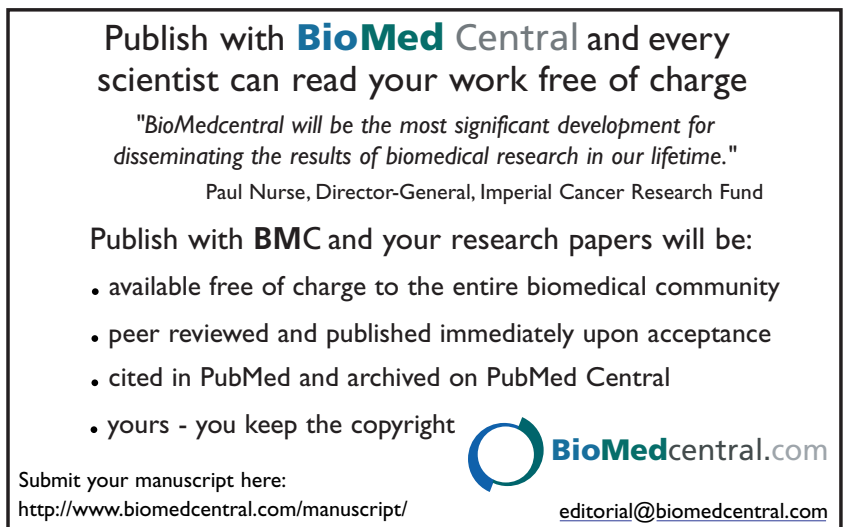

